\title{
Carnosine and Related Peptides: Therapeutic Potential in Age-Related Disorders
}

\author{
José H. Cararoํㅜ, Emilio L. Streck², Patricia F. Schuck ${ }^{1}$, Gustavo da C. Ferreira ${ }^{3, *}$ \\ ${ }^{1}$ Laboratório de Erros Inatos do Metabolismo, Programa de Pós-Graduação em Ciências \\ da Saúde, Universidade do Extremo Sul Catarinense, Criciúma, SC, Brazil \\ ${ }^{2}$ Laboratório de Bioenergética, Programa de Pós-Graduação em Ciências da Saúde, Universidade do \\ Extremo Sul Catarinense, Criciúma, SC, Brazil \\ ${ }^{3}$ Laboratório de Bioenergética, Instituto de Bioquímica Médica Leopoldo de Meis, Universidade Federal \\ do Rio de Janeiro, Rio de Janeiro, RJ, Brazil
}

[Received March 5, 2015; Revised June 10, 2015; Accepted June 16, 2015]

\begin{abstract}
Imidazole dipeptides (ID), such as carnosine ( $\beta$-alanyl-L-histidine), are compounds widely distributed in excitable tissues of vertebrates. ID are also endowed of several biochemical properties in biological tissues, including antioxidant, bivalent metal ion chelating, proton buffering, and carbonyl scavenger activities. Furthermore, remarkable biological effects have been assigned to such compounds in age-related human disorders and in patients whose activity of serum carnosinase is deficient or undetectable. Nevertheless, the precise biological role of ID is still to be unraveled. In the present review we shall discuss some evidences from clinical and basic studies for the utilization of ID as a drug therapy for age-related human disorders.
\end{abstract}

Key words: imidazole dipeptides; biological activity; aging; children; serum carnosinase deficiency

\section{Introduction}

\section{Imidazole dipeptides (ID) in biological tissues}

The mammalian essential amino acid L-histidine originates compounds in biological tissues, including histamine [1], and the ID carnosine, homocarnosine, and anserine [2]. Liquid chromatography/electrospray ionization tandem mass spectrometry analyses performed in plasma or tissue homogenates from rats showed that the highest concentrations of carnosine and anserine are found in skeletal muscles, heart and brain.
Homocarnosine was found only in the brain. No ID were detectable in plasma, liver, kidney and lung [3].

The homocarnosine biosynthesis from L-histidine and $\gamma$ aminobutyric acid (GABA) is favored by a ligase enzyme namely carnosine synthase (EC 6.3.2.11) [2]. Homocarnosine is present in human central nervous system (CNS) and is synthesized in specific GABAergic neurons [4-5]. Anserine is not present in human tissues [6], and it may be isolated from chicken pectoral muscle extracts [7]. This dipeptide is subject to cleavage to $\beta$ alanine and 1-methyl-L-histidine by the human serum 
carnosinase, also known as $\beta$-Ala-His dipeptidase (EC 3.4.13.20) activity [8].

\section{Carnosine}

Carnosine is a naturally occurring dipeptide ( $\beta$-alanine and L-histidine), widely distributed in muscular and nervous tissues of vertebrates, whose concentrations may reach up to $20 \mathrm{mM}$ [9-10]. The non-proteinogenic and precursor amino acid $\beta$-alanine is mainly synthesized by the liver degradation of uracil, from the conversion of $\beta$ ureidopropionate in $\beta$-alanine, ammonia, and carbon dioxide catalyzed by $\beta$-ureidopropionase (EC 3.5.1.6) [11]. Carnosine was first isolated from skeletal muscle extracts in the end of XIX century by Gulewitsch and colleagues [12-3].

Dietary carnosine and anserine uptake is carried out by an intestinal peptide transporter namely human $\mathrm{H}^{+} /$peptide cotransporter 1 (hPEPT1) [14]. Carnosine biosynthesis from its precursor amino acids is catalyzed by carnosine synthase coupled to ATP hydrolysis [2]. It occurs in excitable tissues, such as skeletal muscle cells [15] and oligodendrocytes [16]. On the other hand, the cleavage of carnosine and homocarnosine is carried out by serum carnosinase [8], which is synthesized in brain and liver [17] and secreted into the cerebrospinal fluid (CSF) and blood [18]. Other dipeptidase able to cleave carnosine in biological tissues is the cytosol nonspecific dipeptidase (CNDP, EC 3.4.13.18). This protein is constitutively expressed in brain, kidneys, liver, spleen, gonads, lungs, and pancreas [17]. In the brain, CNDP is synthesized in histaminergic neurons of tuberomammillary nucleus, which is located in hypothalamus [19]. The products of ID hydrolysis are metabolized and excreted in urine [6].

Several biochemical properties have been assigned to ID by researchers since the past century, in animal and cell culture studies. Carnosine is the most studied compound in the group of imidazolic dipeptides and presents putative antioxidant [20], bivalent metal ion chelating [21], radioprotective [22], muscular proton buffering [23], and reactive carbonyl scavenger activities [24]. The latter property was also suggested to the D-enantiomer of carnosine [25].

Furthermore, many physiological effects were associated to carnosine and other peptides. For instance, evidence suggests that carnosine is distributed in dorsal horn of mice spinal cord; and presents antinociceptive activity [26]. Other physiological effect assigned to carnosine is the immunostimulant activity. Thus, Li and co-workers [27] showed that the number of lymphocytes in spleen and the level of plasma corticosterone presented no significant alterations in stressed animals receiving carnosine previously. The administration of this peptide also elicited antidepressant-like effects in rats subjected to forced swimming [28]. Since most of the mechanisms underlying the physiological effects of ID reported hitherto are unknown, the precise role of ID in biological tissues and fluids remains unclear.

\section{Is the biological activity of ID associated to age of the subject?}

It has been suggested that carnosine may be involved in the prevention and therapy of oxidative driven disorders, including neurodegenerative diseases and hypoxicischemic damage of CNS [29]. The major risk factor to neurodegenerative disorders is aging, and mitochondria were implicated in such phenomenon through mechanisms that include accumulation of mutations in mitochondrial DNA (mtDNA) and reactive oxygen species (ROS) generation [30].

Murphey and co-workers [31] reported carnosine accumulation in the serum $(20-30 \mu \mathrm{mol} / \mathrm{mL})$ and urine (60-200 $\mu \mathrm{mol} / 24 \mathrm{~h})$ of patients affected by a rare inherited metabolic disorder - serum carnosinase deficiency (SCD, OMIM 212200). The onset of the symptoms occurred during infancy and children presented progressive mental retardation, an unspecific symptom of such disorder. The present review gather information regarding the current state of ID utilization as drug therapy for age-related human disorders in clinical trials, as well as their safety.

\section{Metabolic changes with aging}

The explanation of the causes of aging remains somewhat controversial. However, the prime candidates seem to be environmental and endogenous factors which affect an organism's ability to survive by trigger genetic changes (e.g., DNA damage and telomere shortening), alteration in gene expression, oxidative stress, impairment of ATP provision, and accumulation of aberrant proteins [32]. According to the Free Radical Theory, aging and related neurodegenerative diseases are mainly assigned to oxidation of cellular components by free radicals, catalyzed by oxidative enzymes and traces of metal ions [33].

Mitochondria are the organelles responsible by ATP generation, $\mathrm{Ca}^{2+}$ uptake and storage, and generation and detoxification of ROS. The mitochondrial membrane potential $\left(\Delta \psi_{\mathrm{m}}\right)$ drives such functions, while many apoptotic signals are triggered by cytochrome $c$ release into the cytoplasm [34]. Bioenergetics dysfunction is a more recent approach that may aid to understand the aging process. Respiratory chain complex I (EC 1.6.5.3) is thought to be mostly affected by oxidative damage, becoming strongly rate limiting for electron transfer [35]. Additionally, it was shown a significant decrease in the 
state 3 of respiration and membrane potential in heart mitochondria of aged rats [36].

The oxidative damage and other age-induced alterations can be mitigated by long-term regimens of caloric restriction. The underlying mechanisms remain unknown, but caloric restriction protective effects may be mediated by the decrease in the mitochondrial proton leak [37], connecting disruption of bioenergetics to aging.

Mutations in mtDNA and decrease in the mtDNA copy number were associated to the pathogenesis of type 2 diabetes [38] linking, in part, this metabolic disorder to aging. However, the age-related energy dysfunction cannot be only attributable to changes in the sequence and copy number of the mtDNA. According to analyses carried out by Bakala and co-workers [39], liver mitochondria of aged rats present a decline in their capacity to generate ATP mainly due to decreased expression of oxidative phosphorylation complexes and glycation damage to $\beta$-oxidation and citric acid/urea cycle enzymes.

The ROS net production by the mitochondria may be the major source of oxidative damage that accumulates with age and it is detrimental to many mitochondrial functions. The contributing factors appear to include the intrinsic rate of proton leakage across the inner mitochondrial membrane, decreased membrane fluidity, and decreased levels and function of the cardiolipin, a membrane phospholipid that supports the functioning of many proteins of the inner mitochondrial membrane [40]. The oxidation of such compound seems to be involved in several age-related mitochondrial bioenergetics changes, such as electron transport chain impairment, substrate transport defects, activation of mitochondrial permeability transition, and cytochrome $c$ release [41].

Other age-related metabolic changes in the lipid metabolism may be triggered. The physiological actions of the circulating hormone leptin decrease with aging, which is associated to inflammation of adipose tissue, decreased thermogenesis, and possibly impaired cognitive function [42]. In this way, aging increases cholesterol trafficking proteins in astrocytes and myelination in hippocampus of F344 rats. In fact, an early-adult metabolic changes favoring lipid/ketone body oxidation may trigger inflammatory degradation of myelin and result in excessive cholesterol that, in turn, activates its transport from astrocytes to remyelinating oligodendrocytes. Such processes may compete with neuronal pathways for bioenergetics supplies, thereby impairing cognitive function [43].

Other molecular and cellular signaling pathways were also suggested to underlie aging process. The nuclear factor- $\kappa \mathrm{B} \quad(\mathrm{NF}-\mathrm{\kappa} \mathrm{B})$ triggers a pro-inflammatory environment with glycolytic metabolism, whereas the silent information regulator 1 (SIRT1) supports oxidative respiration and promotes the resolution of the inflammation. Increased NF- $\kappa \mathrm{B}$ activation and subsequent inflammatory responses disturb bioenergetics driven by SIRT1, which could contribute to the aging process due to the potential generation of several agerelated metabolic disorders, including type 2 diabetes and cardiovascular diseases [44]. Recently, it was proposed that ID might counteract many of the above-mentioned mechanisms associated to aging $[45,50,58]$.

\section{ID and aging: general mechanisms}

The bulk of ID content synthesized in major vertebrates is present in excitable tissues, mainly in skeletal muscle [6]. HPLC analyses showed that the content of carnosine and anserine in skeletal muscle significantly decreases with age in SAMP8 senescence-accelerated mice. Oral creatine supplementation temporarily prevents such decline in muscle ID content and it is associated with attenuated muscle fatigue and enhanced recovery [46]. The proposed mechanisms for ID action include proton buffering [23] and sarcoplasmic reticulum $\mathrm{Ca}^{2+}$-release channels modulation [47-8].

Antioxidant activity was assigned to carnosine [20], whose ability to react with superoxide, hydroxyl [9], and hypochlorite anions in vitro [49] was previously shown. Additionally, such dipeptide inhibits the non-enzymatic glycosylation and protein cross-linking induced by reactive aldehydes (e.g., MDA), the formation of proteinassociated advanced glycosylation end products (AGEs) induced by MDA, and the formation of DNA-protein cross-linking induced by acetaldehyde and formaldehyde in vitro [49]. Carnosine administration $(250 \mathrm{mg} / \mathrm{kg}$, daily) for 1 month to aged rats (22 months old) resulted in significantly decreased levels of MDA and conjugated diene in serum, LDL + VLDL fraction, and erythrocytes, and increased GSH levels in erythrocytes [45].

Methylglyoxal (MG), a product of the spontaneous decomposition of glycolitic trioses phosphate, is a source of glycative damage to protein, mitochondrial dysfunction, and ROS generation. Carnosine reacts with MG and other reactive aldehydes, and scavenges various ROS, inhibiting ischemia and age-related mechanisms [50]. Additionally such dipeptide was showed to suppress the toxicicity of protein carbonyls by reacting to form protein carbonyl-carnosine adducts [51] and inhibit glycation damage mediated by $\mathrm{MG}$ and glucose in Escherichia coli [52]. In fact, the putative anti-aging effect of carnosine seems more readily explainable by its ability to react with protein carbonyls than its antioxidant activity [51].

In attempt to examine the various mechanisms proposed to explain the anti-aging effects assigned to carnosine, studies searched for some of the carnosine 
adducts that have been predicted following the dipeptide's putative reaction with reactive carbonyl compounds [53]. Evidence on quenching mechanism of ID towards $\alpha, \beta$ unsatured aldehydes generated by lipid peroxidation (e.g., 4-hydroxy-trans-2-nonenal and acrolein) suggests that it occurs through a multi-step mechanisms, including: $i$ ) the formation of a reversible imine intermediate; ii) a key intramolecular Michael addiction between the histidinic imidazole ring and the acceptor $\beta$-carbon atom; and (iii) the hydrolysis of the imine group to give a final hemiacetal adduct (for more details, see reference 24). This was demonstrated in vitro through the reaction of carnosine and homocarnosine with acrolein, with further characterization of the formed adducts [54].

Another mechanism attributed to carnosine is modulation of glycolysis and inhibition the glycolysisinduced protein dysfunction. The underlying mechanisms are unclear, but the dipeptide may mimic the action of rapamycin, inhibiting the cellular respiration regulatory complex - mammalian target of rapamycin (mTOR) [55]. Alternatively, carnosine may activate the gluconeogenic enzyme fructose-biphosphatase (FBPase, EC 3.1.3.11), as shown in rabbit muscle [56]. If carnosine activates FBPase in vivo by chelating the allosteric regulator $\mathrm{Zn}^{2+}$ [57], this would create a futile ATP-consuming cycle since the ATP-utilizing enzyme phosphofructokinase-1 (EC 2.7.1.11) converts fructose 6-phosphate into fructose 1,6-bisphosphate. This cycle would decrease ATP levels and synthesis as well as the supply of carbon skeletons for amino acid synthesis, impairing the growth of senescent cells by intensive glycolytic process [58].

Microscopy, flow cytometry, and ELISA analysis performed by Kantha and co-workers [59] showed that high carnosine concentrations $(30 \mathrm{mM})$ significantly reduce the generation of the DNA oxidative damage marker 8-hydroxy-2'-deoxyguanosine in rat embryonic fibroblasts subjected to oxidative damage. Carnosine (20 $\mathrm{mM}$ ) also decreases the shortening rate of telomeres in cultured human fetal lung fibroblasts, possibly preventing these structures from oxidative DNA damage [60]. These findings may aid to explain the anti-aging and lifeextension effects attributed to carnosine, as shown in cultured HFF-1 and MRC-5 human fibroblasts [61-2], mice of SAMP1 strain [63], and male Drosophila melanogaster [64].

\section{ID and age-related human disorders}

\section{Cataract}

According to the World Health Organization (WHO)'s Prevention of Blindness and Visual Impairment, cataract is the clouding of the eye lens, which prevents clear vision. Most cases of such disorder are related to aging process. Cataract is the leading cause of blindness, accounting for $51 \%$ of the world incidence (20 million people approximately) in 2010. The risk factors include smoking, prolonged ultraviolet light (UV) exposure, diabetes mellitus, and high body mass index [65]. Other possible causes include reduced dietary antioxidant intake, severe dehydration, and side effect of drugs such as corticosteroids [66].

Cataract is associated with conformational changes and unfolding of proteins in the lens, which could arise from post-translational modifications induced by the Maillard reaction [67]. This process is defined as a nonenzymatic modification of amino acids by reaction with sugars, cyanide or steroids. The unfolded proteins of the lens are susceptible to oxidative damage, characterized by the linkage of polypeptide chains through disulphide bonds and formation of high molecular weight waterinsoluble aggregates. Such molecules account for the increased scattering of light, resulting in the opacity of the lens typical of the cataract [66].

In this scenario, an in vitro study showed that the dipeptides $N$-acetylcarnosine - a pro-drug version of carnosine ( $N$-acetyl- $\beta$-alanyl-L-histidine) - and anserine, but not carnosine itself, significantly suppressed UVinduced aggregation of the $\beta$-L-crystallin - a protein associated to the lens. An inhibition at the initial stages of protein photochemical damage and a decrease in size of protein aggregates were also detected in the presence of both dipeptides [68]. In vivo and ex vivo studies also showed the antioxidant activity of the carnosine, $\mathrm{N}$ acetylcarnosine, and $\beta$-alanyl-L-histamine. These compounds significantly prevented biological molecules from lipid peroxide-induced damage, including proteins, enzymes, unsaturated fatty acids, and membrane phospholipids, which may occur in the lens cells and predispose to cataract [13]. Importantly, the proposed mechanism of action for $N$-acetylcarnosine is the antilipid peroxidation [66], representing a modified form of carnosine resistant to hydrolysis by the human serum carnosinase [58].

Ophthalmic formulations containing $\mathrm{N}$ acetylcarnosine may be promising for treating many ocular diseases mediated by lipid peroxide- and ROSinduced damage, including cataract [69], since such disorder is associated with the lack of a reductive detoxification system of phospholipid hydroperoxides in the lens cell membranes and biomolecules [70]. Telomere shortening and increased oxidative stress in human lens cells may be the result of the peroxidative damage to such cells, which is associated with the etiology of cataract. Therefore, telomere shortening rate as well as damages to telomere of the lens cells could be reduced by the systemic administration of carnosine (protected from enzyme 
hydrolysis), $N$-acetylcarnosine eye drops, and ophthalmic formulations [71].

By convention, a suitable clinical trial of any drug should be performed with a large number of volunteers and by a long-term as a multicentre, randomized, double blind, and placebo-controlled trial [66]. In spite of the promising evidences, the clinical studies performed to date with $\mathrm{N}$-acetylcarnosine eye drops [72-8] present certain limitations, including a limited number of patients. Further independent research of clinical effectiveness is needed to validate research claims of $\mathrm{N}$-acetylcarnosine that have potential conflicts of interest in humans $[66,79]$.

\section{Alzheimer's disease (AD)}

$\mathrm{AD}$ is a neurodegenerative disease and the most common cause of dementia in elderly [80], characterized by a progressive cognitive decline. This disorder may be triggered by the formation of senile plaques primarily composed by amyloid- $\beta$ peptide $(\mathrm{A} \beta)$, and neurofibrillary tangles mainly composed by hyperphosphorylated tau $(\tau)$ protein in the brain. About $5-10 \%$ of cases are inheritable, occurring in an autosomal-dominant manner. Three proteins are acknowledged be associated with such familial cases: the amyloid precursor protein, which is enzymatically cleaved to produce $\mathrm{A} \beta$, and the presenilins 1 and 2 [30].

As showed in in vitro experiments, $\mathrm{A} \beta$ aggregation may be accelerated by cross-linking mediated by AGEs and carnosine, and other AGE-inhibitors were showed to attenuate this process [81-2]. Recently, a study was performed investigating the effects of carnosine on the formation of peptide fragment $\mathrm{A} \beta 1-42$ aggregates. Atomic force microscopy and thioflavin $\mathrm{T}$ assays showed an inhibition of $\mathrm{A} \beta 1-42$ fibrillogenesis in vitro and differences in the aggregation state of $\mathrm{A} \beta 1-42$ small prefibrillar structures in the presence of carnosine [83], suggesting that carnosine may be effective against the $\mathrm{A} \beta$ aggregation in $\mathrm{AD}$ brain.

Pretreatment of rat brain endothelial cells with carnosine $(20 \mathrm{mM})$ also protected them against the toxicity mediated by an $\mathrm{A} \beta$ peptide fragment (residues 25-35) [49, 82]. Similarly, a set of in vivo experiments showed that $3 \times \mathrm{Tg}-\mathrm{AD}$ mice receiving carnosine orally exhibited strong reduction of the hippocampal intraneuronal accumulation of $\mathrm{A} \beta$ and improvement of mitochondrial dysfunction related to aging and $\mathrm{AD}$ : recovery of the complexes II and IV activities in hippocampus and recovery of the complexes I and IV activities in cerebral cortex [84].

Carbonic anhydrase (CA, EC 4.2.1.1) activity is significantly decreased in the brain of AD patients [85]. In this scenario, kinetic and X-ray crystallographic studies performed by Temperini and co-workers [86] showed that both carnosine and its methyl ester acted as more efficient activators of three human CA isoforms than L-histidine, indicating that carnosine and its derivatives may have some therapeutic potential to mitigate the AD evolution through this mechanism.

Carnosine and homocarnosine present significant quenching ability towards acrolein [54], a lipid peroxidation product that was shown to be increased in $\mathrm{AD}$ brain [87]. The same authors detected different reaction products between acrolein and carnosine/homocarnosine, affording further insights on the involvement of such dipeptides in normal and pathological brain. MDA is also significantly increased in the brain of AD patients [88] and carnosine diminished MDA-induced cell damage, as shown by a significant decrease in protein cross-linking and ROS generation, as well as prevention of the $\Delta \psi_{\mathrm{m}}$ dissipation in cultured rat cortical neurons [89].

In a double-blind placebo-controlled study addressing the therapeutic potential of carnosine in $\mathrm{AD}$, it was assessed the efficacy of a combination of carnosine plus other antioxidants (formula $\mathrm{F}$ ) in volunteers receiving donepezil $(n=26)$, in comparison to patients receiving donepezil plus placebo $(n=26)$ [90]. Using the Mini-Mental State Examination II (MMSE II) score, Cornelli [90] observed a significantly improvement of cognition in the group receiving donepezil plus formula $\mathrm{F}$, as compared to those receiving donepezil plus placebo.

\section{Parkinson's disease (PD)}

PD is a neurodegenerative disorder clinically characterized by progressive rigidity, bradykinesia, and tremor. The pathological findings include loss of pigmented dopaminergic neurons in the substantia nigra and the presence of distinctive cytoplasmic inclusions immunoreactive for $\alpha$-synuclein and ubiquitin, i.e., the Lewy's bodies [30]. It was shown that carnosine, homocarnosine, and anserine significantly inhibited aggregation and carbonylation of $\alpha$-synuclein mediated by ceruloplasmin and $\mathrm{H}_{2} \mathrm{O}_{2}$ in vitro [91].

In PD animal models, carnosine supplementation $(0.5,1$, and $2 \mathrm{~g} / \mathrm{L}$, in drinking water) significantly decreased the oxidative stress, inflammatory damage, and dopamine depletion induced by 1-methyl-4-phenyl1,2,3,6-tetrahydropyridine (MPTP). On the other hand, reduced glutathione (GSH) levels, carnosine content, and mRNA levels and activities of the glutathione peroxidase (EC 1.11.1.9) and superoxide dismutase (EC 1.15.1.1) were found increased in the same animals. Furthermore, it was also found a decrease in the levels of interleukin 6 , tumor necrosis factor- $\alpha, \mathrm{NO}$, as well as the mRNA level and activity of the inducible nitric oxide synthase (EC 
1.14.13.39) in striatum of mice receiving MPTP and supplemented with carnosine [92].

Increased levels of the neurotoxin 1-methyl-6,7dihydroxy-1,2,3,4-tetrahydroisoquinoline (salsolinol) are detected in the CSF of PD patients [93]. An in vitro study showed that carnosine and $\mathrm{N}$-acetylcarnosine prevented salsolinol-mediated oxidative modification of the ferritin, reflected as a decrease in ferritin aggregation and inhibition of the carbonyl and dityrosine formation [94]. This latter finding may be substantiated by the antioxidant [20] and carbonyl-scavenger [24] activities assigned to ID, as previously discussed.

Hyperhomocysteinemia is also present in PD patients [95-6]. Homocysteine activates cells expressing $N$ methyl-D-aspartic acid (NMDA) receptors (e.g., neurons) and elicits $\mathrm{Ca}^{2+}$ and ROS intracellular accumulation [97]. Carnosine significantly prevented the cells expressing NMDA receptors from excitotoxic damage mediated by NMDA and homocysteine [98], and it cannot be ruled out that this effect may be, at least in part, mediated by antioxidant and chelating ability of carnosine towards ROS and $\mathrm{Ca}^{2+}$. Other possible mechanisms for the biological activity of carnosine in PD may be acknowledged in the review performed by Hipkiss and coworkers (2013), which emphasizes the putative role of the MG in the pathogenesis of such disorder. As previously discussed, carnosine counteracts MG and other cytotoxic aldehydes [50], which in part could substantiate its neuroprotective effect in PD patients.

A pilot study performed by Boldyrev and colleagues [99] assessed the efficacy of carnosine $(1.5 \mathrm{~g}$, daily, by 30 days) as a complement to DOPA drug therapy in PD patients. It was concluded that the combination of carnosine with basic therapy for certain PD patients may be a reasonable way to improve the protocol of PD treatment and to decrease the possible toxic effects of the overloading of DOPA-containing drugs [99]. Due to the small number of volunteers and the short duration of this study, a larger and long-term trial is needed to confirm the benefit of carnosine combined to DOPA drug therapy.

\section{Other disturbances potentially age-related}

Apart from cataract and neurodegeneration, research claims on potential age-related conditions against which carnosine could be explored therapeutically include diabetes and diabetic complications, ischemia, osteoporosis, deafness, slow wound healing, hypertension (raised blood pressure), and heart disease. However, it is noteworthy that further studies are required to verify these research claims [32].

Recent HPLC and computational studies performed by Vistoli and co-workers [24] with 40 ID that are more stable to enzyme hydrolysis revealed that the peptides L-
Lysyl-L-Histidine-OMe and L-Tyrosyl-D-Histidine-OMe showed a carbonyl quenching activity slightly weaker, but significantly more selective than that of carnosine. These peptides may represent promising candidates for the design of improved carnosine derivatives.

\section{ID in SCD: evidence that the biological activity of such compounds may be detrimental to younger subjects}

SCD or carnosinemia is an autosomal-recessive inherited metabolic disease with an estimated prevalence of 1:500,000 newborns [100]. The serum carnosinase gene $(C N D P 1)$ is located in the region $18 \mathrm{q} 22.3$ [101]. SCD is biochemically characterized by carnosine accumulation in the serum and urine of affected patients due to low activity of serum carnosinase [31]. On the other hand, serum ID concentration in healthy subjects under fasting is undetectable [102]. The main clinical manifestations assigned to SCD include intellectual deficiency, growth delay, seizures, twitching, lethargy, generalized muscle wasting [31], and hypotonia [103].

To date, the most of the SCD patients reported were children with neurological dysfunction [31, 103-8]. Homocarnosinosis (EC 236130), which was considered as a distinct disorder, may be a severe serum carnosinase deficiency, since the presence of the enzyme homocarnosinase was not shown in human tissues [109]. The phenotype of SCD patients is very variable and it was suggested that the enzyme deficiency is not pivotal to the neurological dysfunction [108, 110].

However, it cannot be ruled out that the ID accumulation in the earlier stages of development of SCD patients may be detrimental to some tissues and organs. In the brain, ID-induced potential damage may aid to explain the neurologic disturbances observed in patients. Since metal ions are related to neurotransmission and synaptic plasticity modulation [111], it would be reasonable to infer that the dysfunction may be assigned to a detrimental chelating activity elicited by increased ID concentration in CNS [21].

Serum carnosinase activity is undetectable in newborns and young children, increasing with age and reaching the adult range at about 10 years [31, 112]. Consequently, carnosine levels in the human skeletal muscle [113], as well as the homocarnosine concentration in human CSF [114] significantly decrease with age. A recent paper published by Macarini and co-workers [115] corroborates such proposition. It was showed a significant decrease in the mitochondrial respiratory chain complexes I-III and II activities in skeletal muscle of young rats (30 days old) receiving carnosine $(100 \mathrm{mg} / \mathrm{kg}$ body weight acutely, as compared to animals receiving vehicle.

Therefore, carnosine accumulation could damage tissues in the earlier stages of development in SCD 
patients and possibly in newborns and children, whose serum carnosinase activity is undetectable or deficient. A putative mechanism whereby the carnosine accumulation could induce damage in tissues is the previously discussed modulation/inhibition of glycolysis. Such process would occur in growing young cells with high demand for ATP and for carbon skeletons to the synthesis of amino acids through this metabolic pathway [58]. Another possibility is the impairment of ID utilization as precursors of GABA, $\beta$-alanine, and L-histidine, which may act as a contributing factor to neurologic dysfunction [116].

\section{Conclusion}

Aging is a physiological process whose precise underlying mechanisms are unclear, although it may include DNA damage and telomere shortening, changes in gene expression, induction of oxidative stress, impairment of ATP synthesis, and accumulation of altered proteins. Many physiological effects observed in experimental studies with cell culture, animals and humans are assigned to ID. In this scenario, carnosine is thought to counteract several mechanisms associated to aging process due to its presumable biochemical properties, including antioxidant, bivalent metal ion chelating, muscular proton buffering, anti cross-linking, and reactive carbonyl scavenger activities.

At present, ID seem to represent potential therapeutic agents in studies performed both in vitro and in vivo. Additionally, preliminary clinical studies with these compounds have also shown promising results, as observed to cataract, $\mathrm{AD}$ and $\mathrm{PD}$, as well as other agerelated human disorders.

Even though only beneficial effects are assigned to carnosine $[99,117]$ and other peptides, it cannot be ruled out that there are patients prone to present supraphysiological and potentially toxic ID concentrations. Therefore, experimental studies are needed to support or refute the presented hypothesis, and possibly its underlying mechanisms. The data raised by these studies may aid to shed new light on the role of ID in biological tissues in an age-specific manner.

\section{Acknowledgements}

Gustavo da Costa Ferreira is supported with personal grant from the Brazilian National Council for Scientific and Technological Development (CNPq) and FAPERJ.

\section{References}

[1] Moya-Garcia AA, Medina MA, Sánchez-Jiménez F (2005). Mammalian histidine decarboxylase: from structure to function. Bioessays, 27:57-63.
[2] Drozak J, Veiga-da-Cunha M, Vertommen D, Stroobant V, Van Schaftingen E (2010). Molecular identification of carnosine synthase as ATP-grasp domain-containing protein 1 (ATPGD1). J Biol Chem, 285:9346-56.

[3] Aldini G, Orioli M, Carini M, Maffei Facino R (2004). Profiling histidine-containing dipeptides in rat tissues by liquid chromatography/electrospray ionization tandem mass spectrometry. J Mass Spectrom, 39:141728.

[4] Kish SJ, Perry TL, Hansen S (1979). Regional distribution of homocarnosine, homocarnosinecarnosine synthetase and homocarnosinase in human brain. J Neurochem, 32:1629-36.

[5] Petroff OAC, Hyder F, Mattson RH, Rothman DL (2001). Topiramate increases human brain GABA within thirty minutes. Proc Intl Soc Mag Reson Med, 9:959.

[6] Boldyrev AA, Severin SE (1990). The histidinecontaining dipeptides, carnosine and anserine: distribution, properties and biological significance. Adv Enzyme Regul, 30:175-94.

[7] Babizhayev MA, Deyev AI, Yegorov YE (2014). LCarnosine modulates respiratory burst and reactive oxygen species production in neutrophil biochemistry and function: may oral dosage form of non-hydrolized dipeptide L-carnosine complement anti-infective antiinfluenza flu treatment, prevention and self-care as an alternative to the conventional vaccination? Curr Clin Pharmacol, 9:93-115.

[8] Jackson MC, Kucera CM, Lenney JF (1991). Purification and properties of human serum carnosinase. Clin Chim Acta, 196:193-205.

[9] Quinn PJ, Boldyrev AA, Formazuyk VE (1992). Carnosine: its properties, functions and potential therapeutic applications. Mol Aspects Med, 13:379444.

[10] Hipkiss AR, Brownson C, Bertani MF, Ruiz E, Ferro A (2002). Reaction of carnosine with aged proteins: another protective process? Ann N Y Acad Sci, 959:285-94.

[11] van Kuilenburg AB, Dobritzsch D, Meijer J, Meinsma $\mathrm{R}$, Benoist JF, Assmann B et al (2010). Dihydropyrimidinase deficiency: Phenotype, genotype and structural consequences in 17 patients. Biochim Biophys Acta, 1802:639-48.

[12] Gulewitsch W, Amiradžibi S (1900). Ueber das carnosin, eine neue organische base des fleischextractes. Ber Dtsch Chem Ges, 33:1902-3.

[13] Babizhayev MA (2006). Biological activities of the natural imidazole-containing peptidomimetics $\mathrm{N}$ acetylcarnosine, carcinine and L-carnosine in ophthalmic and skin care products. Life Sci, 78:234357.

Geissler S, Zwarg M, Knütter I, Markwardt F, Brandsch $M$ (2010). The bioactive dipeptide anserine is transported by human proton-coupled peptide transporters. FEBS J, 277:790-5. 
[15] Bakardjiev A, Bauer K (1994). Transport of $\beta$-alanine and biosynthesis of carnosine by skeletal muscle cells in primary culture. Eur J Biochem, 225:617-23.

[16] Hoffmann AM, Bakardjiev A, Bauer K (1996). Carnosine-synthesis in cultures of rat glial cells is restricted to oligodendrocytes and carnosine uptake to astrocytes. Neurosci Lett, 215:29-32.

[17] Teufel M, Saudek V, Ledig JP, Bernhardt A, Boularand S, Carreau A et al (2003). Sequence identification and characterization of human carnosinase and a closely related non-specific dipeptidase. J Biol Chem, 278:6521-31.

[18] Schoen P, Everts H, de Boer T, van Oeveren W (2003). Serum carnosinase activity in plasma and serum: validation of a method and values in cardiopulmonary bypass surgery. Clin Chem, 49:1930-2.

[19] Otani H, Okumura A, Nagai K, Okumura N (2008). Colocalization of a carnosine-splitting enzyme, tissue carnosinase (CN2)/cytosolic non-specific dipeptidase 2 (CNDP2), with histidine decarboxylase in the tuberomammillary nucleus of the hypothalamus. Neurosci Lett, 445:166-9.

[20] Manhiani PS, Northcutt JK, Han I, Bridges WC, Dawson PL (2013). Antioxidant activity of carnosine extracted from various poultry tissues. Poult Sci, 92:444-53.

[21] Baran EJ (2000). Metal complexes of carnosine. Biochemistry (Mosc), 65:789-97.

[22] Tanaka RA, Ramos FM, Almeida SM, Vizioli MR, Bóscolo FN (2005). Evaluation of radioprotective effect of carnosine ( $\beta$-alanyl-L-histidine) on the wound healing in rats. J Appl Oral Sci, 13:253-8.

[23] Abe H (2000). Role of histidine-related compounds as intracellular proton buffering constituents in vertebrate muscle. Biochemistry (Mosc), 65:757-65.

[24] Vistoli G, De Maddis D, Straniero V, Pedretti A, Pallavicini M, Valoti E et al (2013). Exploring the space of histidine containing dipeptides in search of novel efficient RCS sequestering agents. Eur J Med Chem, 66:153-60.

[25] Aldini G, Orioli M, Rossoni G, Savi F, Braidotti P, Vistoli G et al (2011). The carbonyl scavenger carnosine ameliorates dyslipidaemia and renal function in Zucker obese rats. J Cell Mol Med, 15:1339-54.

[26] Ohsawa M, Mutoh J, Asato M, Yamamoto S, Ono H, Hisa H et al (2012). Carnosine has antinociceptive properties in the inflammation-induced nociceptive response in mice. Eur J Pharmacol, 682:56-61.

[27] Li YF, He RR, Tsoi B, Li XD, Li WX, Abe K et al (2012). Anti-stress effects of carnosine on restraintevoked immunocompromise in mice through spleen lymphocyte number maintenance. PLoS One, 7:e33190.

[28] Tomonaga S, Yamane H, Onitsuka E, Yamada S, Sato M, Takahata Y et al (2008). Carnosine-induced antidepressant-like activity in rats. Pharmacol Biochem Behav, 89:627-32.

[29] Bellia F, Vecchio G, Cuzzocrea S, Calabrese V, Rizzarelli E (2011). Neuroprotective features of carnosine in oxidative driven diseases. Mol Aspects Med, 32:258-66.

[30] Lin MT, Beal MF (2006). Mitochondrial dysfunction and oxidative stress in neurodegenerative diseases. Nature, 443:787-95.

[31] Murphey WH, Lindmark DG, Patchen LI, Housler ME, Harrod EK, Mosovich L (1973). Serum carnosinase deficiency concomitant with mental retardation. Pediatr Res, 7:601-6.

[32] Hipkiss AR (2009). Carnosine and its possible roles in nutrition and health. Adv Food Nutr Res, 57:87-154.

[33] Harman D (1956). Aging: a theory based on free radical and radiation chemistry. J Gerontol, 11:298-300.

[34] Nicholls DG (2004). Mitochondrial membrane potential and aging. Aging Cell, 3:35-40.

[35] Lenaz G, Bovina C, D'Aurelio M, Fato R, Formiggini G, Genova ML et al (2002). Role of mitochondria in oxidative stress and aging. Ann N Y Acad Sci, 959:199213.

[36] Petrosillo G, Matera M, Moro N, Ruggiero FM, Paradies G (2009). Mitochondrial complex I dysfunction in rat heart with aging: critical role of reactive oxygen species and cardiolipin. Free Radic Biol Med, 46:88-94.

[37] Hunt ND, Hyun DH, Allard JS, Minor RK, Mattson MP, Ingram DK et al (2006). Bioenergetics of aging and calorie restriction. Ageing Res Rev, 5:125-43.

[38] Rolo AP, Palmeira CM (2006). Diabetes and mitochondrial function: role of hyperglycemia and oxidative stress. Toxicol Appl Pharmacol, 212:167-78.

[39] Bakala H, Ladouce R, Baraibar MA, Friguet B (2013). Differential expression and glycative damage affect specific mitochondrial proteins with aging in rat liver. Biochim Biophys Acta, 1832:2057-67.

[40] Shigenaga MK, Hagen TM, Ames BN (1994). Oxidative damage and mitochondrial decay in aging. Proc Natl Acad Sci U S A, 91:10771-8.

[41] Paradies G, Petrosillo G, Paradies V, Ruggiero FM (2010). Oxidative stress, mitochondrial bioenergetics, and cardiolipin in aging. Free Radic Biol Med, 48:128695.

[42] Carter S, Caron A, Richard D, Picard F (2013). Role of leptin resistance in the development of obesity in older patients. Clin Interv Aging, 8:829-44.

[43] Kadish I, Thibault O, Blalock EM, Chen KC, Gant JC, Porter NM et al (2009). Hippocampal and cognitive aging across the lifespan: a bioenergetic shift precedes and increased cholesterol trafficking parallels memory impairment. J Neurosci, 29:1805-16.

[44] Kauppinen A, Suuronen T, Ojala J, Kaarniranta K, Salminen A (2013). Antagonistic crosstalk between $\mathrm{NF}-\kappa \mathrm{B}$ and SIRT1 in the regulation of inflammation and metabolic disorders. Cell Signal, 25:1939-48.

[45] Aydín AF, Küskü-Kiraz Z, Doğru-Abbasoğlu S, Uysal M (2010). Effect of carnosine treatment on oxidative stress in serum, apoB-containing lipoproteins fraction and erythrocytes of aged rats. Pharmacol Rep, 62:7339.

[46] Derave W, Jones G, Hespel P, Harris RC (2008). Creatine supplementation augments skeletal muscle 
carnosine content in senescence-accelerated mice (SAMP8). Rejuvenation Res, 11:641-7.

[47] Batrukova MA, Rubtsov AM (1997). Histidinecontaining dipeptides as endogenous regulators of the activity of sarcoplasmic reticulum $\mathrm{Ca}^{2+}$-release channels. Biochim Biophys Acta, 1324:142-50.

[48] Rubtsov AM (2001). Molecular mechanisms of regulation of the activity of sarcoplasmic reticulum $\mathrm{Ca}^{2+}$-release channels (ryanodine receptors), muscle fatigue, and Severin's phenomenon. Biochemistry (Mosc), 66:1132-43.

[49] Hipkiss AR, Preston JE, Himsworth DT, Worthington VC, Keown M, Michaelis J et al (1998). Pluripotent protective effects of carnosine, a naturally occurring dipeptide. Ann N Y Acad Sci, 854:37-53.

[50] Hipkiss AR (2010). Aging, Proteotoxicity, Mitochondria, Glycation, NAD and Carnosine: Possible Inter-Relationships and Resolution of the Oxygen Paradox. Front Aging Neurosci, 2:10.

[51] Hipkiss AR (2000). Carnosine and protein carbonyl groups: a possible relationship. Biochemistry (Mosc), 65:771-8.

[52] Pepper ED, Farrell MJ, Nord G, Finkel SE (2010). Antiglycation effects of carnosine and other compounds on the long-term survival of Escherichia coli. Appl Environ Microbiol, 76:7925-30.

[53] Hipkiss AR (2009). On the enigma of carnosine's antiageing actions. Exp Gerontol, 44:237-42.

[54] Carini M, Aldini G, Beretta G, Arlandini E, Facino RM (2003). Acrolein-sequestering ability of endogenous dipeptides: characterization of carnosine and homocarnosine/acrolein adducts by electrospray ionization tandem mass spectrometry. J Mass Spectrom, 38:996-1006.

[55] Hipkiss AR (2011). Energy metabolism, proteotoxic stress and age-related dysfunction - protection by carnosine. Mol Aspects Med, 32:267-78.

[56] Ikeda T, Kimura K, Hama T, Tamaki N (1980). Activation of rabbit muscle fructose 1,6-bisphosphatase by histidine and carnosine. J Biochem, 87:179-85.

[57] Tejwani GA, Pedrosa FO, Pontremoli S, Horecker BL (1976). Dual role of $\mathrm{Zn}^{2+}$ as inhibitor and activator of fructose 1,6-bisphosphatase of rat liver. Proc Natl Acad Sci U S A, 73:2692-5.

[58] Hipkiss AR, Cartwright SP, Bromley C, Gross SR, Bill RM (2013). Carnosine: can understanding its actions on energy metabolism and protein homeostasis inform its therapeutic potential? Chem Cent J, 7:38.

[59] Kantha SS, Wada S, Tanaka H, Takeuchi M, Watabe S, Ochi H (1996). Carnosine sustains the retention of cell morphology in continuous fibroblast culture subjected to nutritional insult. Biochem Biophys Res Commun, 223:278-82.

[60] Shao L, Li QH, Tan Z (2004). L-Carnosine reduces telomere damage and shortening rate in cultured normal fibroblasts. Biochem Biophys Res Commun, 324:9316.

[61] McFarland GA, Holliday R (1994). Retardation of the senescence of cultured human diploid fibroblasts by carnosine. Exp Cell Res, 212:167-75.
[62] McFarland GA, Holliday R (1999). Further evidence for the rejuvenating effects of the dipeptide L-carnosine on cultured human diploid fibroblasts. Exp Gerontol, 34:35-45.

[63] Gallant S, Semyonova M, Yuneva M (2000). Carnosine as a potential anti-senescence drug. Biochemistry (Mosc), 65:866-8.

[64] Stvolinsky S, Antipin M, Meguro K, Sato T, Abe H, Boldyrev A (2010). Effect of carnosine and its Troloxmodified derivatives on life span of Drosophila melanogaster. Rejuvenation Res, 13:453-7.

[65] World Health Organization (2014). Priority eye diseases: Cataract. Available at: http://www.who.int/blindness/causes/priority/en/ind ex1.html. Access in: January $18^{\text {th }} 2014$.

[66] Toh T, Morton J, Coxon J, Elder MJ (2007). Medical treatment of cataract. Clin Experiment Ophthalmol, 35:664-71.

[67] Crabbe MJ (1998). Cataract as a conformational disease - the Maillard reaction, $\alpha$-crystallin and chemotherapy. Cell Mol Biol (Noisy-le-grand), 44:1047-50.

[68] Dizhevskaya AK, Muranov KO, Boldyrev AA, Ostrovsky MA (2012). Natural dipeptides as minichaperones: molecular mechanism of inhibition of lens $\beta \mathrm{L}$-crystallin aggregation. Curr Aging Sci, 5:236-41.

[69] Babizhayev MA (2011). Mitochondria induce oxidative stress, generation of reactive oxygen species and redox state unbalance of the eye lens leading to human cataract formation: disruption of redox lens organization by phospholipid hydroperoxides as a common basis for cataract disease. Cell Biochem Funct, 29:183-206.

[70] Babizhayev MA (1996). Failure to withstand oxidative stress induced by phospholipid hydroperoxides as a possible cause of the lens opacities in systemic diseases and ageing. Biochim Biophys Acta, 1315:87-99.

[71] Babizhayev MA, Vishnyakova KS, Yegorov YE (2011). Telomere-dependent senescent phenotype of lens epithelial cells as a biological marker of aging and cataractogenesis: the role of oxidative stress intensity and specific mechanism of phospholipid hydroperoxide toxicity in lens and aqueous. Fundam Clin Pharmacol, 25:139-62.

[72] Babizhayev MA (2004). Rejuvenation of visual functions in older adult drivers and drivers with cataract during a short-term administration of $\mathrm{N}$-acetylcarnosine lubricant eye drops. Rejuvenation Res, 7:186-98.

[73] Babizhayev MA, Deyev AI, Yermakova VN, Semiletov YA, Davydova NG, Kurysheva NI et al (2001). NAcetylcarnosine, a natural histidine-containing dipeptide, as a potent ophthalmic drug in treatment of human cataracts. Peptides, 22:979-94.

[74] Babizhayev MA, Deyev AI, Yermakova VN, Semiletov YA, Davydova NG, Doroshenko VS et al (2002). Efficacy of $N$-acetylcarnosine in the treatment of cataracts. Drugs R D, 3:87-103.

[75] Babizhayev MA, Kasus-Jacobi A (2009). State of the art clinical efficacy and safety evaluation of $\mathrm{N}$ acetylcarnosine dipeptide ophthalmic prodrug. 
Principles for the delivery, self-bioactivation, molecular targets and interaction with a highly evolved histidyl-hydrazide structure in the treatment and therapeutic management of a group of sight-threatening eye diseases. Curr Clin Pharmacol, 4:4-37.

[76] Babizhayev MA, Guiotto A, Kasus-Jacobi A (2009). NAcetylcarnosine and histidyl-hydrazide are potent agents for multitargeted ophthalmic therapy of senile cataracts and diabetic ocular complications. J Drug Target, 17:36-63.

[77] Babizhayev MA, Burke L, Micans P, Richer SP (2009). $\mathrm{N}$-Acetylcarnosine sustained drug delivery eye drops to control the signs of ageless vision: glare sensitivity, cataract amelioration and quality of vision currently available treatment for the challenging 50,000-patient population. Clin Interv Aging, 4:31-50.

[78] Babizhayev MA, Micans P, Guiotto A, Kasus-Jacobi A (2009). N-Acetylcarnosine lubricant eyedrops possess all-in-one universal antioxidant protective effects of Lcarnosine in aqueous and lipid membrane environments, aldehyde scavenging, and transglycation activities inherent to cataracts: a clinical study of the new vision-saving drug $\mathrm{N}$-acetylcarnosine eyedrop therapy in a database population of over 50,500 patients. Am J Ther, 16:517-33.

[79] Cloud A, Tandon A, Calhoun J, Cebulla CM (2012). Rapid formation and resolution of cataracts following orthopedic surgery for a patient with Charcot-MarieTooth disease. Arch Ophthalmol, 130:260-2.

[80] Griffin WS (2006). Inflammation and neurodegenerative diseases. Am J Clin Nutr, 83:470S474S.

[81] Münch G, Mayer S, Michaelis J, Hipkiss AR, Riederer $\mathrm{P}$, Müller R et al (1997). Influence of advanced glycation end-products and AGE-inhibitors on nucleation-dependent polymerization of $\beta$-amyloid peptide. Biochim Biophys Acta, 1360:17-29.

[82] Preston JE, Hipkiss AR, Himsworth DT, Romero IA, Abbott JN (1998). Toxic effects of $\beta$-amyloid (25-35) on immortalised rat brain endothelial cell: protection by carnosine, homocarnosine and $\beta$-alanine. Neurosci Lett, 242:105-8.

[83] Aloisi A, Barca A, Romano A, Guerrieri S, Storelli C, Rinaldi R et al (2013). Anti-aggregating effect of the naturally occurring dipeptide carnosine on a $\beta 1-42$ fibril formation. PLoS One, 8:e68159.

[84] Corona C, Frazzini V, Silvestri E, Lattanzio R, La Sorda $\mathrm{R}$, Piantelli M et al (2011). Effects of dietary supplementation of carnosine on mitochondrial dysfunction, amyloid pathology, and cognitive deficits in 3xTg-AD mice. PLoS One, 6:e17971.

[85] Meier-Ruge W, Iwangoff P, Reichlmeier K (1984). Neurochemical enzyme changes in Alzheimer's and Pick's disease. Arch Gerontol Geriatr, 3:161-5.

[86] Temperini C, Scozzafava A, Puccetti L, Supuran CT (2005). Carbonic anhydrase activators: X-ray crystal structure of the adduct of human isozyme II with Lhistidine as a platform for the design of stronger activators. Bioorg Med Chem Lett, 15:5136-41.
[87] Lovell MA, Xie C, Markesbery WR (2001). Acrolein is increased in Alzheimer's disease brain and is toxic to primary hippocampal cultures. Neurobiol Aging, 22:187-94.

[88] Dei R, Takeda A, Niwa H, Li M, Nakagomi Y, Watanabe $\mathrm{M}$ et al (2002). Lipid peroxidation and advanced glycation end products in the brain in normal aging and in Alzheimer's disease. Acta Neuropathol, 104:113-22.

[89] Cheng J, Wang F, Yu DF, Wu PF, Chen JG (2011). The cytotoxic mechanism of malondialdehyde and protective effect of carnosine via protein crosslinking/mitochondrial dysfunction/reactive oxygen species/MAPK pathway in neurons. Eur J Pharmacol, 650:184-94.

[90] Cornelli U (2010). Treatment of Alzheimer's disease with a cholinesterase inhibitor combined with antioxidants. Neurodegener Dis, 7:193-202.

[91] Kim KS, Choi SY, Kwon HY, Won MH, Kang TC, Kang JH (2002). The ceruloplasmin and hydrogen peroxide system induces $\alpha$-synuclein aggregation in vitro. Biochimie, 84:625-31.

[92] Tsai SJ, Kuo WW, Liu WH, Yin MC (2010). Antioxidative and Anti-Inflammatory Protection from Carnosine in the Striatum of MPTP-Treated Mice. J Agric Food Chem, 58:11510-6.

[93] DeCuypere M, Lu Y, Miller DD, LeDoux MS (2008). Regional distribution of tetrahydroisoquinoline derivatives in rodent, human, and Parkinson's disease brain. J Neurochem, 107:1398-413.

[94] Kang JH (2010). Salsolinol, a catechol neurotoxin, induces modification of ferritin: Protection by histidine dipeptide. Environ Toxicol Pharmacol, 29:246-51.

[95] Kuhn W, Roebroek R, Blom H, van Oppenraaij D, Przuntek H, Kretschmer A et al (1998). Elevated plasma levels of homocysteine in Parkinson's disease. Eur Neurol, 40(4):225-7.

[96] Yasui K, Kowa H, Nakaso K, Takeshima T, Nakashima K (2000). Plasma homocysteine and MTHFR C677T genotype in levodopa-treated patients with PD. Neurology, 55:437-40.

[97] Boldyrev AA (2009). Molecular mechanisms of homocysteine toxicity. Biochemistry (Mosc), 74:58998.

[98] Boldyrev A, Bryushkova E, Mashkina A, Vladychenskaya E (2013). Why is homocysteine toxic for the nervous and immune systems? Curr Aging Sci, 6:29-36.

[99] Boldyrev A, Fedorova T, Stepanova M, Dobrotvorskaya I, Kozlova E, Boldanova $\mathrm{N}$ et al (2008). Carnosine [corrected] increases efficiency of DOPA therapy of Parkinson's disease: a pilot study. Rejuvenation Res, 11:821-7.

[100] Vistoli G, Pedretti A, Cattaneo M, Aldini G, Testa B (2006). Homology modeling of human serum carnosinase, a potential medicinal target, and MD simulations of its allosteric activation by citrate. J Med Chem, 49:3269-77.

[101] Janssen B, Hohenadel D, Brinkkoetter P, Peters V, Rind $\mathrm{N}$, Fischer C et al (2005). Carnosine as a protective 
factor in diabetic nephropathy: association with a leucine repeat of the carnosinase gene CNDP1. Diabetes, 54:2320-7.

[102] Pearl PL, Jakobs C, Gibson KM (2007). Disorders of $\beta$ and $\gamma$-amino acids in free and peptide-linked forms. In: Scriver CR, Sly WS, Childs B, editors. The Online Metabolic \& Molecular Bases of Inherited Disease. New York: McGraw- Hill.

[103] Willi SM, Zhang Y, Hill JB, Phelan MC, Michaelis RC, Holden KR (1997). A deletion in the long arm of chromosome 18 in a child with serum carnosinase deficiency. Pediatr Res, 41:210-3.

[104] Perry TL, Hansen S, Tischler B, Bunting R, Berry K (1967). Carnosinemia. A new metabolic disorder associated with neurologic disease and mental defect. $\mathrm{N}$ Engl J Med, 277:1219-27.

[105] van Heeswijk PJ, Trijbels JM, Schretlen ED, van Munster PJ, Monnens LA (1969). A patient with a deficiency of serum-carnosinase activity. Acta Paediatr Scand, 58:584-92.

[106] Wisniewski K, Fleisher L, Rassin D, Lassmann H (1981). Neurological disease in a child with carnosinase deficiency. Neuropediatrics, 12:143-51.

[107] Lunde HA, Gjessing LR, Sjaastad Ø (1986). Homocarnosinosis: influence of dietary restriction of histidine. Neurochem Res, 11:825-38.

[108] Gjessing LR, Lunde HA, Mørkrid L, Lenney JF, Sjaastad $\varnothing$ (1990). Inborn errors of carnosine and homocarnosine metabolism. J Neural Transm Suppl, 29:91-106.

[109] Lenney JF, Peppers SC, Kucera CM, Sjaastad Ø (1983). Homocarnosinosis: lack of serum carnosinase is the defect probably responsible for elevated brain and CSF homocarnosine. Clin Chim Acta, 132:157-65.

[110] Cohen M, Hartlage PL, Krawiecki N, Roesel RA, Carter AL, Hommes FA (1985). Serum carnosinase deficiency: a non-disabling phenotype? J Ment Defic Res, 29:383-9.

[111] Sadiq S, Ghazala Z, Chowdhury A, Büsselberg D (2012). Metal toxicity at the synapse: presynaptic, postsynaptic, and long-term effects. J Toxicol, 2012:132671.

[112] Lenney JF, George RP, Weiss AM, Kucera CM, Chan PW, Rinzler GS (1982). Human serum carnosinase: characterization, distinction from cellular carnosinase, and activation by cadmium. Clin Chim Acta, 123:22131.

[113] Everaert I, Mooyaart A, Baguet A, Zutinic A, Baelde H, Achten E et al (2011). Vegetarianism, female gender and increasing age, but not CNDP1 genotype, are associated with reduced muscle carnosine levels in humans. Amino Acids, 40:1221-9.

[114] Jansen EE, Gibson KM, Shigematsu Y, Jakobs C, Verhoeven NM (2006). A novel, quantitative assay for homocarnosine in cerebrospinal fluid using stableisotope dilution liquid chromatography-tandem mass spectrometry. J Chromatogr B Analyt Technol Biomed Life Sci, 830:196-200.

[115] Macarini JR, Maravai SG, Cararo JH, Dimer NW, Gonçalves CL, Kist LW et al (2014). Impairment of electron transfer chain induced by acute carnosine administration in skeletal muscle of young rats. Biomed Res Int, 2014:632986.

[116] Boldyrev AA, Aldini G, Derave W (2013). Physiology and pathophysiology of carnosine. Physiol Rev, 93:1803-45.

[117] Boldyrev AA, Stvolinsky SL, Fedorova TN, Suslina ZA (2010). Carnosine as a natural antioxidant and geroprotector: from molecular mechanisms to clinical trials. Rejuvenation Res, 13:156-8. 